\title{
Discontinuing Voriconazole Induced No Completely Regression of Multiple Cutaneous Squamous Cell Carcinoma in A Hematopoietic Stem Cell Transplant Patient
}

\author{
Hirakawa $Y^{1^{*}}$, Okuno $A^{1}$, Tanabe $A^{2}$, Okada $M^{3}$, Anzai $N^{3}$, Yamamoto $Y^{4}$, Furukawa $F^{1,5^{*}}$ \\ Departments of Dermatology ${ }^{1}$, Plastic Surgery ${ }^{2}$ and Hematology and Oncology ${ }^{3}$, Takatsuki Red Cross Hospital, Osaka \\ Japan \\ Departments of Dermatology ${ }^{4}$ and Forensic Medicine ${ }^{5}$, Wakayama Medical University, Wakayama, Japan \\ Correspondence: Yuka Hirakawa, M.D., Department of Dermatology, Takatsuki Red Cross Hospital, 1-1-1 Abuno, \\ Takatsuki, Osaka 569-1096, Japan, E-mail: der068@osaka-med.ac.jp
}

\begin{abstract}
Voriconazole is a universal anti-fungal prophylaxis, which is frequently taken to the patients after hematopoietic stem cell transplantation and solid organ transplantation. Voriconazole can cause phototoxicity, multiple erythema in sun-exposed areas may develop actinic keratosis and cutaneous squamous cell carcinoma (cSCC) while taking voriconazole. In North America and Europe, case reports of phototoxicity and aggressive cSCC in patients on voriconazole have been documented. Also 4 cases of voriconazole-associated cSCC have recently been reported in Japan. We describe a Japanese woman with multiple cSCC associated with recurrence of cSCC after discontinuing voriconazole.
\end{abstract}

Keywords: Multiple Cutaneous Squamous Cell Carcinoma; Voriconazole; Hematopoietic Stem Cell Transplantation; Reccurence; Sun-exposed Areas

\section{Introduction}

It is now established that voriconazole is an independent risk factor for the development of cutaneous malignancy in organ transplant recipients ${ }^{[1-3]}$. Recently, a restrospective study from the Mayo Clinic confirmed the association between voriconazole and cutaneous squamous cell carcinoma (cSCC) also after allogeneic hematopoietic stem cell transplantation (allo-HSCT) and identified cumulative days of voriconazole as a risk factor for $\mathrm{cSCC}^{[4]}$ ). We report here a cord blood umbilical transplant recipient who developed multiple cSCC in sun-exposed areas in the setting of voriconazole-induced photodysplasia.

\section{Case Report}

A 73-year-old Japanese woman with a history of recurrent follicular lymphoma underwent allogeneic umbilical cord blood transplantation in January 2013.

She first suffered from follicular lymphoma at the age of 65 with recurrence at 66 and 68 . The patient had no family history. She had one total body irradiation (TBI) treatment before the transplantation. Immediately after the transplantation, the patient was treated with immunosuppression regimen consisting of prednisone and tacrolimus and she had taken these immunosuppressive therapies with chronic graft versus host disease (GVHD) for 1 year. Shereceived prophylactic voriconazole at 200mg twice daily for Aspergillus bronchial colonization from February 2014 to October 2017. In 21 months after continuing voriconazole, photosensitivity and lentigines appeared in sun-exposed areas. She had no photosensitivity in the past. In October 2017, her transplantation team consulted her to the dermatology clinic to evaluate multiple erythema keratosis in sun-exposed areas and a large, growing lesion of her left cheek (Figure 1-a, b). We found a large one on her left cheek with a diameter of $1.0 \mathrm{~cm}$ (Figure 1-c). The skin biopsy 
results showed well differentiated $\mathrm{cSCC}$ (Figure 2-a) and it was excised with a $1 \mathrm{~cm}$ margin along with the underlying periosteum. She discontinued voriconazole at that time. After that, the keratinaization of the face and the backs of the hands frequently occurred (Figure 1-d). After starting her on 5-fluorouracil (5-FU) topical cream, although the keratinizing erythema in part of the fingers disappeared, the lesion on the hand rapidly increased (Figure 1-e).

When we resected 11 lesions surgically, 9 sites were cSCC invasive. The lesion on the left hand was poorly differentiated cSCC (Figure 2-b) and invaded the proper plantar digital nerve of the second finger, she received radiation treatments. 3 weeks later, a new lesion on the left forehead and a recurrent lesion on the left hand appeared. The skin biopsy results also showed poor differentiated cSCC. The left hand was amputated. Afterward, she has not presented with lymphadenopathy and distant metastasis on positron emission tomography (PET)/computed tomography (CT) scan imaging. However, new lesions still in sun-exposed areas appeared one year after discontinuing voriconazole.

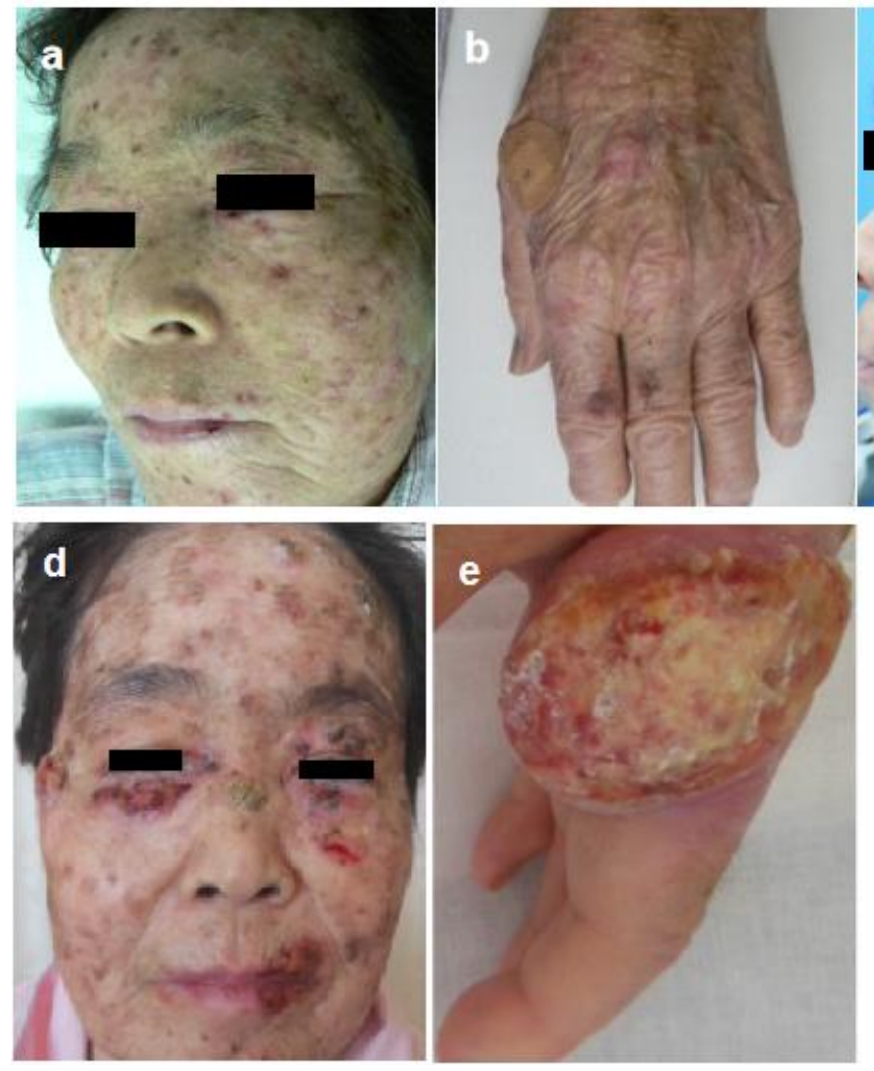

Figure 1; Clinical features. ( $a, b)$ multiple erythema on the face and the backs of the hands. (c) a cutaneous squamous cell carcinoma on the left cheek. (d) multiple cutaneous squamous cell carcinomas appeared on the face. (e) inspite of topical 5-FU, multiple cutaneous squamous cell carcinoma appeared on the left hand rapidly increased.
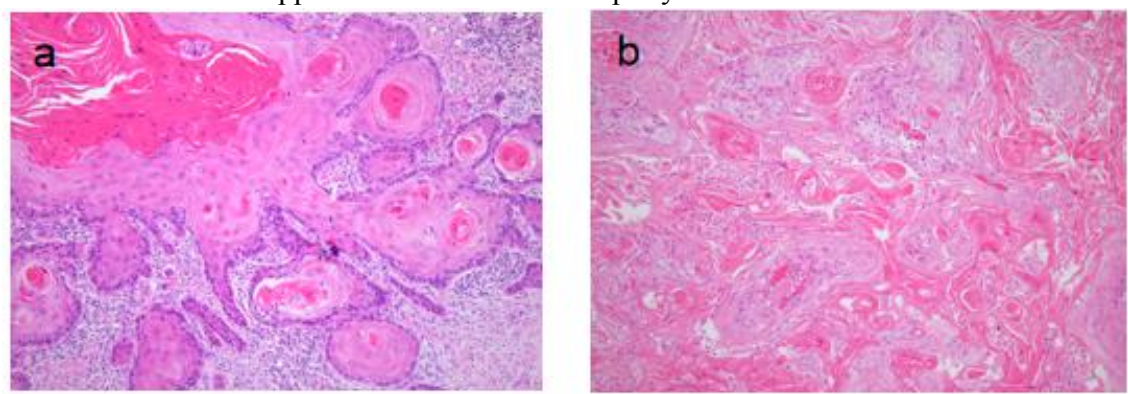

Figure 2; Histological features. (a) well-differentiated cutaneous squamous cell carcinoma on the left cheek (HE staining $\times 400$ ). (b) poor-differentiated cutaneous squamous cell carcinoma on the left hand (HE staining $\times 400$ ).

\section{Discussion}

Voriconazole therapy is often required for extended periods, in particular, patients with ongoing immune system 
dysfunction such as allo-HSCT recipients and SOT recipients. It has been reported that the number of patients with onset multiple erythema and pigments in sun-exposed areas have increased, and they become AK or cSCC while taking voriconazole. The mechanisms by which voriconazole leads to the development of phototoxicity are not clearly understood. As one possibility, it has been suspected that voriconazole induced phototoxicity may be linked to the subsequent development of skin cancer and primary metabolite voriconazole $\mathrm{N}$-oxide may be involved $\mathrm{d}^{[5,6]}$ ). The association was first reported in late 2007, with further cases being reported in bone marrow and solid organ transplant recipients, as well as those with the human immunodeficiency virus $\left.{ }^{[1]}\right)$.

Epaulard et al. have reported the dramatic regression of preneoplastic lesions within several months after discontinuing voriconazole from 4 cases $^{[3]}$ ). In Japan, 4 cases of voriconazole-associated SCC have recently been reported. These lesions of 2 patients were almost clearance after discontinuing voriconazole, on the other hand, those of the other patienst were recurrent and the other patient was aggressive $\left.{ }^{[6]}\right)$. Our patient also still appeared one year after discontinuing voriconazole.

A cohort study from Bridgam and Women's Hospital reported that the patients with 2 to $9 \mathrm{cSCCs}$ have a higher local recurrence rate and nodal metastasis than patients with $1 \mathrm{cSCC}$, and markedly the recurrence rate of the patients with 10 or more cSCCs was much higher in the accumulation results of 10 years ${ }^{[7]}$ ). It is considered that the carcinogenesis of the multiple cSCC may include other multiple factors, the pathogenesis may be different from the general cSCC. Many studies reported that mutagenesis after TB ${ }^{[8]}$ ), chronic inflammation with $\mathrm{GVHD}^{[8]}$ ), mutations by UV radiation ${ }^{[0]}$, Human papilloma virus (HPV), or other as yet uncharacterized viruses ${ }^{[10]}$ ), the effects of carcinogenicity and cytotoxicity from immunosuppressants ${ }^{[11-13]}$ ) and cell adhesion molecules ${ }^{[14]}$ ) may cause multiple cSCC or high risk cSCC. Previous studies have also found both protective and deleterious associations between different HLA types and risk of cSCC in organ transplant recipients. Expression of HLA-A3, -A11, -B27, -DR1, and -DR7 has been suggested to increase risk of non-melanoma skin cancer (NMSC) in some studies ${ }^{[15]}$ ). The patient has HLA-A24, -A26, -B62, -B52, -DR12, and -DR15, those types are not grouped into the risk of cSCC.

Taken together, the lesions of our patient occured in only sun-exposed areas, which considers that these multiple factors trigger skin lesions and UV exposure and voriconazole may promote the development of multiple cSCC. Even though the lesions are in situ, that has the high risk to invasive much early, which is important to remove and treat these lesions as far as possible and the dermatologists should continue dermatologic management of HSCT and SOT recipients carefully.

\title{
Conflict of interest
}

\author{
None declared
}

\section{Referrence}

1. McCarthy KL, Playford EG, Looke DFM, et al. Severevphotosensitivity causing multifocal squamous cell carcinomas secondary to prolonged voriconazole therapy. Clin Infect Dis. 2007; 44: e55-56. Doi:10.1086/511685

2. Vanacker A, Fabré G, Van Dorpe J, et al. Aggressive cutaneous squamous cell carcinoma associated with prolonged voriconazole therapy in a renal transplant patient. Am J Transplant. 2008; 8: 877-880.

Doi : 10.1111/j.1600-6143. 2007. 02140. x

3. Epaulard O, Saint-Raymond C, Villier C, et al. Multiple aggressive squamous cell carcinomas associated with prolonged voriconazole therapy in four immunocompromised patients. Clin Microbilol Infect. 2010; 16(9): 1362-1364 Doi: 10.1111/j.1469-0691.2009.03124.x

4. Wojenski DJ, Bartoo GT, Merten JA, et al. Voriconazole exposure and the risk of cutaneous squamous cell carcinoma in allogeneic hematopoietic stem cell transplant patients. Transpl Infect Dis. 2015; 17(2): 250-258. Doi: 10.1111/tid.12367.

5. Vadnerkar A, Nguyen MH, Mitsani D, et al. Voriconazole exposure and geographic location are independent risk factors for squamous cell carcinoma of the skin among lung transplant recipients. J Heart Lung Transplant. 2010; 29: 1240-1244. Doi: 10.1016/j.healun. 2010.05.022.

6. Wingard JR, Carter SL, Walsh TJ, et al. Randomised, double-blind trial of fluconazole versus voriconazole for prevention of invasive fungal infection after allogeneic hematopoietic cell transplantation.

Blood.2010;116:5111-5118 Doi: 10.1182/blood-2010-02-268151. 
7. Levine DE, Karia PS, Schumults CD. Outcomes of patients with multiple cutaneous squamous cell carcinomas: A 10-Year Single-Institution Cohort Study. JAMA Dermatol.2015;151(11):1220-1225 Doi:

10.1001/jamadermatol.2015.1702.

8. Gmeinhart B, Hinterberger W, Greinix HT, et al.Anaplastic squamous cell carcinoma (SCC) in a patient with chronic cutaneous graft-versus-host disease (GVHD). Bone Marrow Transplant. 1999;23(11):1197-1199 Doi: 10.1038/sj.bmt.1701772

9. Connnolly K, Manders P, Earls P, Epstein RJ. Papillomavirus-associated squamous skin cancers following transplant immunosuppression: one Notch closer to control. Cancer Treat Rev. 2014; 40(2): 205-214 Doi: 10.1016/j.ctrv.2013.08.005.

10. Meyer T, Arndt R, Nindl I, et al. Association of human papillomavirus infections with cutaneous tumors in immunosuppressed patients. Transpl Int. 2003;16(3):146-153 Doi: 10.1007/s00147-002-0525-7

11. Jiyad Z, Olsen CM, Burke MT, et al. Azathioprine and risk of skin cancer in organ transplant recipients: systematic review and meta-analysis. Am J Transplant 2016; 16: 3490-3503 Doi: 10.1111/ajt.13863.

12. O’Neil JO, Edwards LB, Taylor DO. Mycophenolate mofetil and risk of developing malignancy after orthotopic heart transplantation: analysis of the transplant registry of the International Society for Heart and Lung Transplantation. J Heart Lung Transplant 2006; 25: 1186-1191 Doi: 10.1016/j.healun.2006.06.010

13. Coghill AE, Johnson LG, Berg D, et al. Immunosuppressive medications and squamous cell skin carcinoma: nested case-control study within the Skin cancer after Organ Transplant (SCOT) cohort. Am J Transplant 2016; 16: 565-573 Doi: 10.1111/ajt.13596.

14. Furukawa F, Fujii K, Horiguchi Y, et al. Roles of E- and P-cadherin in the human skin. Microsc Res Tech 1997; 38 : 343-352 Doi: 10.1002/(SICI)1097-0029(19970815)38:4<343::AID-JEMT2>3.0.CO;2-K

15. Ingvar Å, Ekström Smedby K, Lindelöf B, et al. No association between infections, HLA type and other transplant-related factors and risk of cutaneous squamous cell carcinoma in solid organ transplant recipients. Acta Derm Venereol 2012; 92(6): 609-614 Doi: 10.2340/00015555-1271. 